\title{
BRILL-NOETHER THEORY FOR MODULI SPACES OF SHEAVES ON ALGEBRAIC VARIETIES
}

\author{
L. COSTA*, R.M. MIRÓ-ROIG**
}

\begin{abstract}
Let $X$ be a smooth projective variety of dimension $n$ and let $H$ be an ample line bundle on $X$. Let $M_{X, H}\left(r ; c_{1}, \cdots, c_{s}\right)$ be the moduli space of $H$-stable vector bundles $E$ on $X$ of rank $r$ and Chern classes $c_{i}(E)=c_{i}$ for $i=1, \cdots, s:=\min \{r, n\}$. We define the Brill-Noether filtration on $M_{X, H}\left(r ; c_{1}, \cdots, c_{s}\right)$ as $W_{H}^{k}\left(r ; c_{1}, \cdots, c_{s}\right)=$ $\left\{E \in M_{X, H}\left(r ; c_{1}, \cdots, c_{s}\right) \mid h^{0}(X, E) \geq k\right\}$ and we realize $W_{H}^{k}\left(r ; c_{1}, \cdots, c_{s}\right)$ as the $k$ th determinantal variety of a morphism of vector bundles on $M_{X, H}\left(r ; c_{1}, \cdots, c_{s}\right)$, provided $H^{i}(E)=0$ for $i \geq 2$ and $E \in M_{X, H}\left(r ; c_{1}, \cdots, c_{s}\right)$. We also compute the expected dimension of $W_{H}^{k}\left(r ; c_{1}, \cdots, c_{s}\right)$. Very surprisingly we will see that the Brill-Noether stratification allow us to compare moduli spaces of vector bundles on Hirzebruch surfaces stables with respect to different polarizations. We will also study the Brill-Noether loci of the moduli space of instanton bundles and we will see that they have the expected dimension.
\end{abstract}

\section{COnTEnts}

1. Introduction

2. Construction of the Brill-Noether Loci

3. Brill-Noether loci and change of polarizations

4. Brill-Noether loci and Instanton bundles

References

\section{INTRODUCTION}

Let $X$ be a smooth projective variety of dimension $n$ over an algebraically closed field $K$ of characteristic 0 and let $M_{X, H}\left(r ; c_{1}, \cdots, c_{s}\right)$ be the moduli space of rank $r$, vector bundles $E$ on $X$ stable with respect to an ample line bundle $H$ and with fixed Chern classes $c_{i}(E)=c_{i}$ for $i=1, \cdots, s:=\min \{r, n\}$. Moduli spaces of stable vector bundles were constructed in the 1970's by Maruyama and since then they have been extensively studied from the point of view of algebraic geometry, of topology and of differential geometry; giving very pleasant connections between these areas. Unfortunately, except in the classical case of vector bundles on curves, relatively little is known about their geometry in terms of the existence and structure of their subvarieties.

Date: October 28, 2018.

1991 Mathematics Subject Classification. 14F05.

Key words and phrases. Brill-Noether, moduli spaces, stability, vector bundles.

* Partially supported by MTM2007-61104.

** Partially supported by MTM2007-61104. 
In the case of line bundles on smooth projective curves $C$ of genus $g$, where the moduli spaces $\operatorname{Pic}^{d}(C)$ of degree $d$ line bundles are all isomorphic to the Jacobian, Brill-Noether theory has long provided a basic source of geometrical information. The classical theory of Brill-Noether has a long history and it is concerned with the subvarieties $W^{k}$ of $P i c^{d}(C)$ whose line bundles have at least $k+1$ independent global sections. Basic questions concerning non-emptiness, connectedness, irreducibility, dimension, singularities, cohomological classes etc ... have been answered when the curve $C$ is generic on the moduli space $M_{g}$ of curves of genus $g$. There are several natural ways to generalize the classical theory of Brill-Noether. First, instead of studying line bundles on curves, we can consider vector bundles of any rank giving rise to the Brill-Noether loci in the moduli space of stable rank $r$ vector bundles on curves studied by Newstead, Teixidor and others. Indeed, during the last two decades, a great amount of job has been made around the Brill-Noether stratification of the moduli space of degree $d$, stable, rank $r$ vector bundles on algebraic curves, giving rise to nice and interesting descriptions of these subvarieties. Nevertheless, it should be mentioned that in spite of big efforts, many questions concerning their geometry still remain open. Second, instead of studying line bundles on curves, we can consider line bundles on varieties of arbitrary dimension and, finally, we can go in both directions simultaneously. We can consider a smooth projective variety $X$ of dimension $n$, an ample line bundle $H$ on $X$, the moduli space $M_{X, H}\left(r ; c_{1}, \cdots, c_{s}\right)$ of rank $r, H$-stable vector bundles $E$ on $X$ with fixed Chern classes $c_{i}(E)=c_{i} ; 1 \leq i \leq \min \{r, n\}$, and we can study the subschemes in $M_{X, H}\left(r ; c_{1}, \cdots, c_{s}\right)$ defined by conditions $\left\{\operatorname{dim} H^{j}(X, E) \geq n_{j}\right\}$. In [11], Gottsche and Hirschowitz studied the Brill-Noether loci in the moduli space of stable vector bundles on $\mathbb{P}^{2}$ and in [15]-[16], Leyenson studied the Brill-Noether loci in the moduli space of stable vector bundles on K3 surfaces. The goal of this paper is to introduce a Brill-Noether theory for moduli spaces of rank $r, H$-stable vector bundles on algebraic varieties of arbitrary dimension, extending, in particular, all the above results to higher dimensional varieties. Once we will have constructed the Brill-Noether stratification in this much more general context, we will address the main problems and we will analyze them for several concrete moduli problems.

Next we outline the structure of this paper. In section 2, we will define the BrillNoether locus $W_{H}^{k}\left(r ; c_{1}, \cdots, c_{s}\right)$ in $M_{X, H}\left(r ; c_{1}, \cdots, c_{s}\right)$ as the set of vector bundles in $M_{X, H}\left(r ; c_{1}, \cdots, c_{s}\right)$ having at least $k$ independent sections and associated to this locus we consider the generalized Brill-Noether number $\rho_{H}^{k}\left(r ; c_{1}, \cdots, c_{s}\right)$. We prove that $W_{H}^{k}\left(r ; c_{1}, \cdots, c_{s}\right)$ has a natural structure of algebraic variety and that any of its nonempty components has dimension $\geq \rho_{H}^{k}\left(r ; c_{1}, \cdots, c_{s}\right)$. Therefore, it is natural to ask whether the numerical condition $\rho_{H}^{k}\left(r ; c_{1}, \cdots, c_{s}\right)<0$ implies that $W_{H}^{k}\left(r ; c_{1}, \cdots, c_{s}\right)$ is empty, and whether $\rho_{H}^{k}\left(r ; c_{1}, \cdots, c_{s}\right) \geq 0$ implies that $W_{H}^{k}\left(r ; c_{1}, \cdots, c_{s}\right)$ is non-empty of dimension $\rho_{H}^{k}\left(r ; c_{1}, \cdots, c_{s}\right)$. We end the section giving examples of situations where the expected dimension $\rho_{H}^{k}\left(r ; c_{1}, \cdots, c_{s}\right)<0$ and $W_{H}^{k}\left(r ; c_{1}, \cdots, c_{s}\right)$ is non-empty; and examples where $\rho_{H}^{k}\left(r ; c_{1}, \cdots, c_{s}\right)>0$ and $W_{H}^{k}\left(r ; c_{1}, \cdots, c_{s}\right)$ is non-empty of dimension strictly greater than $\rho_{H}^{k}\left(r ; c_{1}, \cdots, c_{s}\right)$, in contrast with the classical case. In section 3 , we will analyze how the Brill-Noether stratification will allow us to compare moduli spaces of vector bundles on a smooth projective surface stable with respect to different polarizations. It turns out that the ample cone of a smooth projective surface $X$ has a chamber structure 
such that the moduli space $M_{X, H}\left(r ; c_{1}, c_{2}\right)$ only depends on the chamber of $H$ and the problem consists on determining how the moduli space changes when the polarization crosses a wall between two chambers (see Definition 3.2). Very surprisingly we will realize that in many situations, the Brill Noether locus controls these changes. As a by-product we will obtain a huge family of examples where the dimension of the Brill-Noether loci coincide with the expected one. In the last section, we describe the Brill-Noether loci in the moduli space of mathematical instanton bundles.

Notation: We will work over an algebraically closed field $K$ of characteristic zero. Let $X$ be a smooth projective variety of dimension $n$ and let $E$ be a rank $r$ vector bundle on $X$ with Chern classes $c_{i}(E)=c_{i}, 1 \leq i \leq s:=\min \{r, n\}$. Set $\chi\left(r ; c_{1}, \cdots, c_{s}\right):=\chi(E)$. We will write $h^{i}(E)$ (resp. $\operatorname{ext}^{i}(E, F)$ ) to denote the dimension of the $i$-th cohomology group $H^{i}(X, E)=H^{i}(E)$ (resp. $i$-th Ext group $\operatorname{Ext}^{i}(E, F)$ ) as a $K$-vector space. The sheaf $K_{X}$ will denote the canonical sheaf on $X$.

\section{Construction of the Brill-Noether Loci}

The goal of this section is to prove the existence of a Brill-Noether type stratification on the moduli space of stable vector bundles on smooth projective varieties analogous to the classical stratification of the Picard variety $\operatorname{Pic}^{d}(C)$ of degree $d$ line bundles on a smooth projective curve $C$.

Let us start fixing the notation and some basic definitions. We consider $X$ an $n$ dimensional smooth projective variety, $H$ an ample divisor on $X, r \geq 2$ an integer and $c_{i} \in H^{2 i}(X, \mathbb{Z})$ for $i=1, \cdots, s=\min \{r, n\}$. We denote by $M_{H}=M_{X, H}\left(r ; c_{1}, \cdots, c_{s}\right)$ the moduli space of rank $r$, vector bundles $E$ on $X$, with fixed Chern classes $c_{i}(E)=c_{i}$ for $i=1, \cdots, s$, and $H$-stable according to the following definition due to Mumford and Takemoto.

Definition 2.1. Let $H$ be an ample line bundle on a smooth projective $n$ dimensional variety $X$. For a torsion free sheaf $F$ on $X$ we set

$$
\mu(F)=\mu_{H}(F):=\frac{c_{1}(F) H^{n-1}}{r k(F)} .
$$

The sheaf $F$ is said to be $H$-semistable if

$$
\mu_{H}(E) \leq \mu_{H}(F)
$$

for all non-zero subsheaves $E \subset F$ with $r k(E)<r k(F)$; if strict inequality holds then $F$ is $H$-stable. Notice that for rank $r$ vector bundles $F$ on $X$ with $\left(c_{1}(F) H^{n-1}, r\right)=1$, the concepts of $H$-stability and $H$-semistability coincide.

Remark 2.2. The definition of stability depends on the choice of the ample line bundle $H$. The changes of the moduli space that occur when the line bundle $H$ varies have been studied by several people in great detail and reveals interesting phenomena (see for instance [19]; 6]; 7]; 8]; 9] and references therein). In section 3, we will see how the Brill-Noether loci allow us to study these changes. 
The main goal of this section is to construct a subvariety $W_{H}^{k}\left(r ; c_{1}, \cdots, c_{s}\right)$ of $M_{H}$ whose support is the set of rank $r, H$-stable vector bundles $E$ on $X$ with Chern classes $c_{i}$ such that $h^{0}(E) \geq k$. In other words, we are going to construct a variety

$$
W_{H}^{k}\left(r ; c_{1}, \cdots, c_{s}\right)
$$

such that

$$
\operatorname{Supp}\left(W_{H}^{k}\left(r ; c_{1}, \cdots, c_{s}\right)\right)=\left\{E \in M_{H} \mid h^{0}(E) \geq k\right\}
$$

To achieve our propose, we first need to recall the definition of $k$-th determinantal variety. Let $\phi: E \rightarrow F$ be a morphism between locally free sheaves of ranks $e$, $f$ over an algebraic variety $X$. Upon choosing local trivializations of $E$ and $F$ over an open set $U \subset X$, the morphism $\phi$ is represented by an $e \times f$ matrix $A$ of holomorphic functions. We denote by $U_{k}$ the subset of $U$ whose ideal is generated by the $(k+1) \times(k+1)$ minors of $A$. It is easy to see that $U_{k}$ does not depend on the choice of the trivialization, and therefore there is a well-defined subvariety $X_{k}(\phi)$ of $X$ such that

$$
X_{k}(\phi) \cap U=U_{k}
$$

for every open set $U \subset X$. The variety $X_{k}(\phi)$ is called the $k$-th determinantal variety or the $k$-th degeneracy locus of $\phi$; it is supported on the set

$$
\left\{p \in X \mid r k\left(\phi_{p}\right) \leq k\right\}
$$

and it is clear from the definition that $X_{k}(\phi)$ has codimension at most $(e-k)(f-k)$ when it is non-empty and

$$
X_{k}(\phi) \subset \operatorname{Sing}\left(X_{k+1}(\phi)\right)
$$

whenever $X_{k+1}(\phi) \neq X$.

We are now ready to define the Brill-Noether filtration of $M_{X, H}\left(r ; c_{1}, \cdots, c_{s}\right)$ and to give a formula for the expected dimension of the Brill-Noether locus.

Theorem 2.3. Let $X$ be a smooth projective variety of dimension $n$ and consider a moduli space $M_{H}=M_{X, H}\left(r ; c_{1}, \cdots, c_{s}\right)$ of rank $r, H$-stable vector bundles $E$ on $X$ with fixed Chern classes $c_{i}(E)=c_{i}$. Assume that for any $E \in M_{H}, H^{i}(E)=0$ for $i \geq 2$. Then, for any $k \geq 0$, there exists a determinantal variety $W_{H}^{k}\left(r ; c_{1}, \cdots, c_{s}\right)$ such that

$$
\operatorname{Supp}\left(W_{H}^{k}\left(r ; c_{1}, \cdots, c_{s}\right)\right)=\left\{E \in M_{H} \mid h^{0}(E) \geq k\right\} .
$$

Moreover, each non-empty irreducible component of $W_{H}^{k}\left(r ; c_{1}, \cdots, c_{s}\right)$ has dimension at least

$$
\operatorname{dim}\left(M_{H}\right)-k\left(k-\chi\left(r ; c_{1}, \cdots, c_{s}\right)\right)
$$

and

$$
W_{H}^{k+1}\left(r ; c_{1}, \cdots, c_{s}\right) \subset \operatorname{Sing}\left(W_{H}^{k}\left(r ; c_{1}, \cdots, c_{s}\right)\right)
$$

whenever $W_{H}^{k}\left(r ; c_{1}, \cdots, c_{s}\right) \neq M_{X, H}\left(r ; c_{1}, \cdots, c_{s}\right)$. 
Proof. First of all assume that $M_{H}$ is a fine moduli space. Let $\mathcal{U} \rightarrow X \times M_{H}$ be a universal family such that for any $t \in M_{H},\left.\mathcal{U}\right|_{X \times\{t\}}=E_{t}$ is an $H$-stable rank $r$ vector bundle on $X$ with Chern classes $c_{i}\left(E_{t}\right)=c_{i}$. Let $D$ be an effective divisor on $X$ such that for any $t \in M_{H}$,

$$
h^{0}\left(E_{t}(D)\right)=\chi\left(E_{t}(D)\right), \quad H^{i}\left(E_{t}(D)\right)=0, \quad i \geq 1 .
$$

Consider $\mathcal{D}=D \times M_{H}$ the corresponding product divisor on $X \times M_{H}$ and denote by

$$
\nu: X \times M_{H} \rightarrow M_{H}
$$

the natural projection. It follows from (2.1) and the base change theorem that $\nu_{*} \mathcal{U}(\mathcal{D})$ is a locally free sheaf of rank $\chi\left(E_{t}(D)\right)$ on $M_{H}$ and

$$
R^{i} \nu_{*} \mathcal{U}(\mathcal{D})=0, \quad i>0 .
$$

Therefore, applying the functor $\nu_{*}$ to the short exact sequence

$$
0 \rightarrow \mathcal{U} \rightarrow \mathcal{U}(\mathcal{D}) \rightarrow \mathcal{U}(\mathcal{D}) / \mathcal{U} \rightarrow 0
$$

we get the following exact sequence

$$
0 \rightarrow \nu_{*} \mathcal{U} \rightarrow \nu_{*} \mathcal{U}(\mathcal{D}) \stackrel{\gamma}{\longrightarrow} \nu_{*}(\mathcal{U}(\mathcal{D}) / \mathcal{U}) \rightarrow R^{1} \nu_{*} \mathcal{U} \rightarrow 0
$$

The map $\gamma$ is a morphism between locally free sheaves on $M_{H}$ of rank $\chi\left(E_{t}(D)\right)$ and $\chi\left(E_{t}(D)\right)-\chi(E)$ respectively and the $\left(\chi\left(E_{t}(D)\right)-k\right)$-th determinantal variety

$$
W_{H}^{k}\left(r ; c_{1}, \cdots, c_{s}\right) \subset M_{H}
$$

associated to it has support

$$
\left\{E_{t} \in M_{H} \mid \operatorname{rank} \gamma_{E_{t}} \leq \chi\left(E_{t}(D)\right)-k\right\}
$$

i.e. $W_{H}^{k}\left(r ; c_{1}, \cdots, c_{s}\right)$ is the locus where the fiber of $R^{1} \nu_{*} \mathcal{U}$ has dimension at least $\left(\chi\left(E_{t}(D)\right)-\chi\left(E_{t}\right)\right)-\left(\chi\left(E_{t}(D)\right)-k\right)=k-\chi\left(E_{t}\right)$. For any $E_{t} \in M_{H}$ the assumption $h^{i}\left(E_{t}\right)=0, i \geq 2$, implies

$$
h^{1}\left(E_{t}\right)=h^{0}\left(E_{t}\right)-\chi\left(E_{t}\right) .
$$

Thus,

$$
\begin{aligned}
\operatorname{Supp}\left(W_{H}^{k}\left(r ; c_{1}, \cdots, c_{s}\right)\right) & =\left\{E \in M_{H} \mid h^{1}(E) \geq k-\chi(E)\right\} \\
& =\left\{E \in M_{H} \mid h^{0}(E) \geq k\right\} .
\end{aligned}
$$

Using the language of Fitting ideals, we have that $W_{H}^{k}\left(r ; c_{1}, \cdots, c_{s}\right)$ is the subvariety of $M_{H}$ defined by the $\left(k-\chi\left(E_{t}\right)\right)$ th Fitting ideal of $R^{1} \nu_{*} \mathcal{U}$. Moreover, it can also be seen that $W_{H}^{k}\left(r ; c_{1}, \cdots, c_{s}\right)$ represents the functor

$$
S \rightarrow\left\{\begin{array}{l}
\text { equivalence classes of families } \mathcal{F} \text { on } S \times M_{H} \stackrel{\nu}{\longrightarrow} M_{H} \\
\text { of } H \text {-stable rank } r \text { vector bundles } E \text { on } S \text { with } c_{i}(E)=c_{i} \\
\text { such that the Fitting rank of } R^{1} \nu_{*} \mathcal{F} \text { is at least }(k-\chi(E))
\end{array}\right\} .
$$

Finally, since $W_{H}^{k}\left(r ; c_{1}, \cdots, c_{s}\right)$ is a $\left(\chi\left(E_{t}(D)\right)-k\right)$-determinantal variety associated to a morphism between locally free sheaves of rank $\chi\left(E_{t}(D)\right)$ and $\chi\left(E_{t}(D)\right)-\chi(E)$ respectively, any of its non-empty irreducible components has dimension greater or equal to $\operatorname{dim}\left(M_{H}\right)-$ $k(k-\chi(E))$ and

$$
W_{H}^{k+1}\left(r ; c_{1}, \cdots, c_{s}\right) \subset \operatorname{Sing}\left(W_{H}^{k}\left(r ; c_{1}, \cdots, c_{s}\right)\right)
$$

whenever $W_{H}^{k}\left(r ; c_{1}, \cdots, c_{s}\right) \neq M_{X, H}\left(r ; c_{1}, \cdots, c_{s}\right)$. 
If $M_{H}$ is not a fine moduli space, it is also possible to carry out the construction of the Brill-Noether locus using only the local existence of a universal sheaf on $M_{H}$. Indeed, we carry out the constructions locally, we show the independence of the choice of the locally universal sheaf and we conclude that the construction glue as a global algebraic object.

Remark 2.4. (1) The cohomological assumptions in Theorem 2.3 are natural if we want to have a filtration of the moduli space $M_{H}$ by the subvarieties $W_{H}^{k}\left(r ; c_{1}, \cdots, c_{s}\right)$. Indeed, if $X$ is an $n$-dimensional projective variety, then any vector bundle $E$ on $X$ has $n+1$ cohomological groups whose dimensions are related by Riemann-Roch theorem and one is forced to look for a multigraded filtration of the moduli space $M_{H}$ by means of the sets $\left\{E \in M_{H} \mid h^{i}(E) \geq k_{i}\right\}$. Under the assumptions of Theorem 2.3, $h^{i}(E)=0$ for $i \geq 2$ and for any $E \in M_{H}$, the only non-vanishing cohomology groups are $H^{0}(E)$ and $H^{1}(E)$ and their dimensions are subject to one relation given by Riemann-Roch theorem: $\operatorname{dim} H^{0}(E)-\operatorname{dim} H^{1}(E)=\chi(E)=\chi\left(r ; c_{1}, \cdots, c_{s}\right)$. Hence, it makes sense to consider only the filtration of the moduli space $M_{H}$ by the dimension of the space of global sections.

(2) We want to point out that there exists plenty of vector bundles satisfying the cohomological conditions of Theorem 2.3. For instance, instanton bundles on $\mathbb{P}^{2 n+1}$, Schwarzenberger bundles on $\mathbb{P}^{n}$, Steiner bundles on $\mathbb{P}^{n}$, Steiner and Spinor bundles on a hyperquadric $Q_{n} \subset \mathbb{P}^{n+1}$, etc.

Definition 2.5. The variety $W_{H}^{k}\left(r ; c_{1}, \cdots, c_{s}\right)$ is called the $k$-Brill-Noether locus of the moduli space $M_{H}$ (or simply Brill-Noether locus if there is no confusion) and

$$
\rho_{H}^{k}\left(r ; c_{1}, \cdots, c_{s}\right):=\operatorname{dim} M_{H}-k\left(k-\chi\left(r ; c_{1}, \cdots, c_{s}\right)\right)
$$

is called the generalized Brill-Noether number.

By Theorem 2.3, the Brill-Noether locus $W_{H}^{k}\left(r ; c_{1}, \cdots, c_{s}\right)$ has dimension greater or equal to $\rho_{H}^{k}\left(r ; c_{1}, \cdots, c_{s}\right)$ and the number $\rho_{H}^{k}\left(r ; c_{1}, \cdots, c_{s}\right)$ is also called the expected dimension of the corresponding Brill-Noether locus. Hence, we are led to pose the question whether the dimension of the Brill-Noether locus $W_{H}^{k}\left(r ; c_{1}, \cdots, c_{s}\right)$ and its expected dimension coincide provided the Brill-Noether locus $W_{H}^{k}\left(r ; c_{1}, \cdots, c_{s}\right)$ is non-empty.

Notation 2.6. If there is no confusion then, we will simply write $W^{k}$ and $\rho^{k}$ instead of $W_{H}^{k}\left(r ; c_{1}, \cdots, c_{s}\right)$ and $\rho_{H}^{k}\left(r ; c_{1}, \cdots, c_{s}\right)$.

We will say that the Brill-Noether locus is defined in the moduli space $M_{H}$ whenever the assumptions of Theorem 2.3 are satisfied, i.e. for any $E \in M_{H}, H^{i}(E)=0$ for $i \geq 2$.

Remark 2.7. Notice that when $X$ is a smooth projective curve and we consider the moduli space $P i c^{d}(X)$ of degree $d$ line bundles on $X$, then we recover the classical BrillNoether loci which have been well known since the last century, and the generalized BrillNoether number is the classical Brill-Noether number $\rho=\rho(g, r, d)=g-(r+1)(g-d+r)$.

Corollary 2.8. Let $X$ be a smooth projective surface and let $M_{H}=M_{X, H}\left(r ; c_{1}, c_{2}\right)$ be a moduli space of rank $r, H$-stable vector bundles $E$ on $X$ with fixed Chern classes $c_{i}(E)=$ $c_{i}$. Assume that $c_{1} H \geq r K_{X} H$. Then, for any $k \geq 0$, there exists a determinantal variety $W_{H}^{k}\left(r ; c_{1}, c_{2}\right)$ such that

$$
\operatorname{Supp}\left(W_{H}^{k}\left(r ; c_{1}, c_{2}\right)\right)=\left\{E \in M_{H} \mid h^{0}(E) \geq k\right\} .
$$


Moreover, each non-empty irreducible component of $W_{H}^{k}\left(r ; c_{1}, c_{2}\right)$ has dimension greater or equal to

$$
\rho_{H}^{k}\left(r ; c_{1}, c_{2}\right)=\operatorname{dim}\left(M_{H}\right)-k\left(k-r\left(1+P_{a}(X)\right)+\frac{c_{1} K_{X}}{2}-\frac{c_{1}^{2}}{2}+c_{2}\right)
$$

and $W_{H}^{k+1}\left(r ; c_{1}, c_{2}\right) \subset \operatorname{Sing}\left(W_{H}^{k}\left(r ; c_{1}, c_{2}\right)\right)$ whenever $W_{H}^{k}\left(r ; c_{1}, c_{2}\right) \neq M_{X, H}\left(r ; c_{1}, c_{2}\right)$.

Proof. First of all notice that for any $E \in M_{H}$, the numerical condition $c_{1}(E) H>r K_{X} H$ implies that $H^{2}(E)=0$. Indeed, by Serre duality we have:

$$
H^{2}(E) \cong H^{0}\left(E^{*}\left(K_{X}\right)\right)
$$

and since $E$ is an $H$-stable vector bundle on $X, E^{*}$ is also $H$-stable. Thus, if $H^{2}(E) \neq 0$, $\mathcal{O}_{X}\left(-K_{X}\right) \hookrightarrow E^{*}$ and since $E^{*}$ is $H$-stable we get

$$
\left(-K_{X} H\right)<\frac{c_{1}\left(E^{*}\right) H}{r}=-\frac{c_{1}(E) H}{r}
$$

which contradicts the assumption $c_{1}(E) H \geq r K_{X} H$. Hence the result follows from Theorem 2.3 and the fact that by the Riemann-Roch theorem

$$
\chi\left(r ; c_{1}, c_{2}\right)=r\left(1+P_{a}(X)\right)-\frac{c_{1} K_{X}}{2}+\frac{c_{1}^{2}}{2}-c_{2} .
$$

For any sheaf $E$ on $\mathbb{P}^{2}$, denote by $\chi^{+}=\chi^{+}(E):=\max \{\chi(E), 0\}$. In [11], Göttsche and Hirschowitz gave, under the assumption $c_{1}>-3 r$, a lower bound for the codimension of the Brill-Noether loci $W^{\chi^{+}+1}\left(r ; c_{1}, c_{2}\right)$ of rank $r$, stable vector bundles $E$ on $\mathbb{P}^{2}$ with fixed Chern classes $c_{i}(E)=c_{i}$ such that $h^{0}(E) \geq \chi^{+}+1$. From the previous result we get the following upper bound:

Corollary 2.9. Let $W^{\chi^{+}+1}\left(r ; c_{1}, c_{2}\right)$ be the Brill-Noether locus of rank $r$, stable vector bundles $E$ on $\mathbb{P}^{2}$ with fixed Chern classes $c_{i}(E)=c_{i}$ such that $h^{0}(E) \geq \chi^{+}+1$. Assume that $c_{1}>-3 r$. Then, the following holds:

(a) If $\chi^{+}=\chi\left(r ; c_{1}, c_{2}\right)>0$

$$
2 \leq \operatorname{codim}\left(W^{\chi^{+}+1}\left(r ; c_{1}, c_{2}\right)\right) \leq \chi\left(r ; c_{1}, c_{2}\right)+1 .
$$

(b) If $\chi^{+}=\chi\left(r ; c_{1}, c_{2}\right)=0$

$$
\operatorname{codim}\left(W^{\chi^{+}+1}\left(r ; c_{1}, c_{2}\right)\right)=1
$$

(c) If $\chi^{+}=0>\chi\left(r ; c_{1}, c_{2}\right)$

$$
\operatorname{codim}\left(W^{\chi^{+}+1}\left(r ; c_{1}, c_{2}\right)\right) \leq\left(\chi^{+}+1\right)\left(\chi^{+}+1-\chi\left(r ; c_{1}, c_{2}\right)\right) .
$$

Proof. The lower bounds follow from [11]; Theorem 1. Since $K_{\mathbb{P}^{2}}=\mathcal{O}_{\mathbb{P}^{2}}(-3)$, the hypothesis $c_{1}>-3 r$ is equivalent to $c_{1}(E) H>r K_{X} H$ and the upper bounds follow from Corollary 2.8. 
In subsequent sections we will see that there are plenty of situations where the assumptions of Theorem 2.3 are satisfied and we will prove that in several of them the Brill-Noether loci have exactly the expected dimension, showing that the bound given in Theorem 2.3 is sharp.

Once we have proved the existence of these varieties, it is natural to ask whether the condition $\rho_{H}^{k}\left(r ; c_{1}, \cdots, c_{s}\right)<0$ implies that the variety $W_{H}^{k}\left(r ; c_{1}, \cdots, c_{s}\right)$ is empty and whether the condition $\rho_{H}^{k}\left(r ; c_{1}, \cdots, c_{s}\right) \geq 0$ implies that the variety $W_{H}^{k}\left(r ; c_{1}, \cdots, c_{s}\right)$ is non-empty. Indeed we are led to pose the following three questions.

Question 2.10. Let $X$ be a smooth projective variety of dimension $n$. We consider a moduli space $M_{H}\left(r ; c_{1}, \cdots, c_{s}\right)$ of rank $r, H$-stable vector bundles on $X$ where the BrillNoether locus is defined.

(1) Whether $\rho_{H}^{k}\left(r ; c_{1}, \cdots, c_{s}\right)<0$ implies $W_{H}^{k}\left(r ; c_{1}, \cdots, c_{s}\right)=\emptyset$ ?

(2) Whether $\rho_{H}^{k}\left(r ; c_{1}, \cdots, c_{s}\right) \geq 0$ implies $W_{H}^{k}\left(r ; c_{1}, \cdots, c_{s}\right) \neq \emptyset$ ?

(3) Whether $\rho_{H}^{k}\left(r ; c_{1}, \cdots, c_{s}\right) \geq 0$ and $W_{H}^{k}\left(r ; c_{1}, \cdots, c_{s}\right) \neq \emptyset$ implies

$$
\rho_{H}^{k}\left(r ; c_{1}, \cdots, c_{s}\right)=\operatorname{dim} W_{H}^{k}\left(r ; c_{1}, \cdots, c_{s}\right) \quad ?
$$

If $C$ is a smooth algebraic curve and the moduli space is the Picard variety $\operatorname{Pic}(C)$ of degree $d$ line bundles on $C$, the answer to Question 2.10 is well-known. In fact, classical Brill-Noether theory has its roots dating more than a century ago and it is concerned with the subvarieties of the Picard variety $\operatorname{Pic}^{d}(C)$ determined by degree $d$ line bundles on $C$ having at least a specified number of independent sections. Basic questions, concerning non-emptiness, connectedness, irreducibility, dimension, singularities, cohomology classes, etc ... have been completely answered when the underlying curve is a generic curve in the moduli space $M_{g}$ of curves of genus $g$. Indeed, the Brill-Noether locus is non-empty if $\rho \geq 0$ and connected if $\rho>0$. For a generic curve in $M_{g}$, the Brill-Noether locus is empty if $\rho<0$ and is irreducible of dimension $\rho$ if $\rho>0$. Modern proofs of these results have been given by Kempf, Kleiman and Laksov, Fulton and Lazarsfeld, Griffiths and Harris, and Gieseker and a full treatment of this is contained in [1. The Brill-Noether loci in the moduli space of vector bundles of higher rank on a generic curve $C$ has been studied by Teixidor and others in a series of papers in 1994-2007.

Next example shows that if we deal with algebraic varieties of dimension greater than one, Question 2.10 (1) is no longer true; it gives an example of negative generalized BrillNoether number and the corresponding Brill-Noether locus is non-empty, in contrast with the classical case. Even more, we give examples where the expected dimension of the BrillNoether locus and the dimension of the Brill-Noether locus do not coincide in spite of being positive the generalized Brill-Noether number. Indeed, we have

Example 2.11. Let $X=\mathbb{P}^{1} \times \mathbb{P}^{1}$ be a quadric surface in $\mathbb{P}^{3}$. We denote by $l_{1}, l_{2}$ the generators of $\operatorname{Pic}(X)$ and for any integer $n \geq 2$ we fix the ample line bundle $L=l_{1}+n l_{2}$. We will describe the Brill-Noether stratification of $M_{X, L}\left(2 ;(2 n-1) l_{2}, 2 n\right)$. Indeed, since $(2 n-1) l_{2} L=2 n-1>-4 n-4=2 K_{X} L$, its existence is guaranteed by Corollary 2.8. Let us now prove that for any integer $n \geq 2$ and any integer $j, 0 \leq j \leq n$, the Brill-Noether locus $W_{L}^{j}\left(2 ;(2 n-1) l_{2}, 2 n\right)$ is non-empty. To this end, we consider $\mathcal{F}$ the irreducible family 
parameterizing rank two vector bundles $E$ on $X$ given by an exact sequence

$$
0 \rightarrow \mathcal{O}_{X} \rightarrow E \rightarrow \mathcal{O}_{X}\left((2 n-1) l_{2}\right) \otimes I_{Z} \rightarrow 0
$$

where $Z$ is a locally complete intersection 0 -dimensional scheme of length $2 n$ such that $H^{0} I_{Z}\left((2 n-1) l_{2}\right)=0$.

Notice that since $|Z|=2 n$ and $h^{0} \mathcal{O}_{X}\left((2 n-1) l_{2}\right)=2 n$, the condition

$$
H^{0} I_{Z}\left((2 n-1) l_{2}\right)=0
$$

is satisfied for all generic $Z \in H_{i l b}^{2 n}(X)$ and $\mathcal{F}$ is non-empty. In addition, it can be seen that $\operatorname{dim} \mathcal{F}=4(2 n)-3$ and that $\mathcal{F} \hookrightarrow M_{X, L}\left(2 ;(2 n-1) l_{2}, 2 n\right)$ (see [9]; Proposition 4.6).

Claim 1: $W_{L}^{1}\left(2 ;(2 n-1) l_{2}, 2 n\right) \cong \mathcal{F}$.

Proof of Claim 1: Any $E \in \mathcal{F}$ is $L$-stable and since $H^{0} I_{Z}\left((2 n-1) l_{2}\right)=0$, we have $h^{0}(E)=1$. Thus $\mathcal{F} \subset W_{L}^{1}\left(2 ;(2 n-1) l_{2}, 2 n\right)$. Let us prove the converse. We take a vector bundle $E \in W_{L}^{1}\left(2 ;(2 n-1) l_{2}, 2 n\right)$ and a non-zero global section $s$ of $E$. We denote by $Y$ its scheme of zeros and by $D$ the maximal effective divisor contained in $Y$. Then $s$ can be regarded as a section of $E(-D)$ and its scheme of zeros has codimension $\geq 2$. Thus, for some effective divisor $D=a l_{1}+b l_{2}$ we have a short exact sequence

$$
0 \rightarrow \mathcal{O}_{X}(D) \rightarrow E \rightarrow \mathcal{O}_{X}\left((2 n-1) l_{2}-D\right) \otimes I_{Z} \rightarrow 0
$$

where $Z$ is a locally complete intersection 0 -cycle. Since $D$ is effective, $a, b \geq 0$ and by the $L$-stability of $E$ we have

$$
\left(a l_{1}+b l_{2}\right) L=(a n+b)<\frac{2 n-1}{2}=\frac{c_{1}(E) L}{2} .
$$

Therefore, $a=b=0$ and in fact $E \in \mathcal{F}$.

It follows from Claim 1 that the Brill-Noether locus $W_{L}^{1}\left(2 ;(2 n-1) l_{2}, 2 n\right)$ is an irreducible variety of dimension $8 n-3$ and notice that in this case, its dimension coincides with the expected one. Indeed,

$$
\begin{aligned}
\rho_{L}^{1}\left(2 ;(2 n-1) l_{2}, 2 n\right) & =\operatorname{dim} M_{X, L}\left(2 ;(2 n-1) l_{2}, 2 n\right)-1\left(1-\chi\left(2 ;(2 n-1) l_{2}, 2 n\right)\right) \\
& =8 n-3
\end{aligned}
$$

since by Riemann-Roch theorem,

$$
\chi\left(2 ;(2 n-1) l_{2}, 2 n\right)=2+\frac{\left((2 n-1) l_{2}\right)\left(2 l_{1}+2 l_{2}\right)}{2}+\frac{\left((2 n-1) l_{2}\right)^{2}}{2}-2 n=1 .
$$

For any $i, 1 \leq i \leq n-1$, we can choose a 0 -dimensional scheme $Z_{i}$ on $X$ of length $2 n$ such that $h^{0}\left(I_{Z_{i}}\left((2 n-1) l_{2}\right)\right)=i$ and $h^{0}\left(I_{Z_{i}}\left((2 n-i-1) l_{2}\right)\right)=0$. Thus, if we denote by $E_{i}$ the rank two vector bundle on $X$ given by the exact sequence

$$
0 \rightarrow \mathcal{O}_{X} \rightarrow E_{i} \rightarrow \mathcal{O}_{X}\left((2 n-1) l_{2}\right) \otimes I_{Z_{i}} \rightarrow 0
$$

we have $h^{0}\left(E_{i}\right)=i+1$.

Claim 2: $E_{i}$ is $L$-stable.

Proof of Claim 2: Since $E_{i}$ is given by a non-trivial extension

$$
0 \rightarrow \mathcal{O}_{X} \rightarrow E_{i} \rightarrow \mathcal{O}_{X}\left((2 n-1) l_{2}\right) \otimes I_{Z_{i}} \rightarrow 0,
$$


given a sub-line bundle $\mathcal{O}_{X}\left(a l_{1}+b l_{2}\right)$ of $E_{i}$ we have two possible cases:

$$
\begin{gathered}
(1) \quad \mathcal{O}_{X}\left(a l_{1}+b l_{2}\right) \hookrightarrow \mathcal{O}_{X} \\
(2) \quad \mathcal{O}_{X}\left(a l_{1}+b l_{2}\right) \hookrightarrow \mathcal{O}_{X}\left((2 n-1) l_{2}\right) \otimes I_{Z_{i}} .
\end{gathered}
$$

In case $(1),\left(a l_{1}+b l_{2}\right) L \leq 0<\frac{2 n-1}{2}=\frac{c_{1}\left(E_{i}\right) L}{2}$. In case $(2),-a l_{1}+(2 n-1-b) l_{2}$ is an effective divisor and hence $a \leq 0$ and $b \leq 2 n-1$. On the other hand, since

$$
H^{0}\left(\mathcal{O}_{X}\left(a l_{1}+(b-i) l_{2}\right)\right) \subset H^{0}\left(I_{Z_{i}}\left((2 n-i-1) l_{2}\right)\right)=0
$$

we have $a<0$ or $b<i$. If $b<i$, since $a \leq 0$ and $i \leq n-1$, we have

$$
\left(a l_{1}+b l_{2}\right) L=a n+b \leq n-1<\frac{2 n-1}{2}=\frac{c_{1}\left(E_{i}\right) L}{2} .
$$

Assume $a<0$. In that case, since $b \leq 2 n-1$ we get

$$
\left(a l_{1}+b l_{2}\right) L=a n+b \leq-n+b \leq n-1<\frac{2 n-1}{2}=\frac{c_{1}\left(E_{i}\right) L}{2} .
$$

Therefore, $E_{i}$ is $L$-stable which proves Claim 2. loci

By Claim 2, $E_{i} \in W_{L}^{i+1}\left(2 ;(2 n-1) l_{2}, 2 n\right)$ and we get a chain of non-empty Brill-Noether

$$
\begin{array}{r}
M_{X, L}\left(2 ;(2 n-1) l_{2}, 2 n\right) \supset W_{L}^{1}\left(2 ;(2 n-1) l_{2}, 2 n\right) \supset W_{L}^{2}\left(2 ;(2 n-1) l_{2}, 2 n\right) \supset \cdots \\
\cdots \supset W_{L}^{n}\left(2 ;(2 n-1) l_{2}, 2 n\right) \supsetneq \emptyset .
\end{array}
$$

Notice that for any $k, 1 \leq k \leq n$, such that $8 n-3<k(k-1)$ the Brill-Noether locus $W_{L}^{k}\left(2 ;(2 n-1) l_{2}, 2 n\right)$ is non-empty and the generalized Brill-Noether number, $\rho_{L}^{k}(2 ;(2 n-$ 1) $\left.l_{2}, 2 n\right)$, is negative. In fact,

$$
\begin{aligned}
\rho_{L}^{k}\left(2 ;(2 n-1) l_{2}, 2 n\right) & =\operatorname{dim} M_{X, L}\left(2 ;(2 n-1) l_{2}, 2 n\right)-k\left(k-\chi\left(2 ;(2 n-1) l_{2}, 2 n\right)\right) \\
& =8 n-3-k(k-1)
\end{aligned}
$$

where the last equality follows from Proposition 3.6 and the equation (2.4).

Finally, we have that for any $k$ such that $2<k(k-1)<8 n-3$, the generalized Brill-Noether number, $\rho_{L}^{k}\left(2 ;(2 n-1) l_{2}, 2 n\right)$, is positive; however, the Brill-Noether locus $W_{L}^{k}\left(2 ;(2 n-1) l_{2}, 2 n\right)$ is non-empty and its dimension is greater than the expected one. In fact, it is enough to observe that

$$
\operatorname{dim} W_{L}^{k}\left(2 ;(2 n-1) l_{2}, 2 n\right)=8 n-2 k-1>\rho_{L}^{k}\left(2 ;(2 n-1) l_{2}, 2 n\right) .
$$

\section{Brill-Noether loci AND Change of polarizations}

In this section we will see that the Brill-Noether stratification allow us to compare moduli spaces of vector bundles on smooth projective surfaces, stable with respect to different polarizations.

Let $X$ be a smooth projective variety of dimension $n$. As we pointed out in Remark 2.2 , the notion of stability of vector bundles on $X$ strongly depends on the ample divisor. Hence it is natural to consider the following interesting problem: 
Problem 3.1. What is the difference between the moduli spaces

$$
M_{H}=M_{X, H}\left(r ; c_{1}, \cdots, c_{\min \{r, n\}}\right) \quad \text { and } \quad M_{L}=M_{X, L}\left(r ; c_{1}, \cdots, c_{\min \{r, n\}}\right)
$$

where $H$ and $L$ are two different polarizations?

It turns out that the ample cone of $X$ has a chamber structure such that the moduli space $M_{X, H}\left(r ; c_{1}, \cdots, c_{\min \{r, n\}}\right)$ only depends on the chamber of $H$ and the problem consists on determining how the moduli space changes when the polarization crosses a wall between two chambers (see Definition 3.2). These changes have been explicitly described in very few occasions (see for instance [6], [8], [9]).

We will focus our attention in case where $X$ is a Hirzebruch surface and we will deal with stable rank two vector bundles on $X$. Very surprisingly we will realize that in a huge family of moduli spaces, the difference between two moduli spaces $M_{L}\left(2 ; c_{1}, c_{2}\right)$ and $M_{H}\left(2 ; c_{1}, c_{2}\right)$ (where $L$ and $H$ are two polarizations sitting in chambers sharing a common wall), is precisely described by suitable Brill-Noether loci. Moreover, we will be able to compute the dimension of these Brill-Noether loci and we will prove that the expected dimension of these Brill-Noether loci is indeed the dimension.

To start with, let us recall the basic results about walls and chambers due to Qin ([19]).

Definition 3.2. (i) Let $\xi \in N u m(X) \otimes \mathbb{R}$. We define

$$
\mathcal{W}^{\xi}:=C_{X} \cap\{x \in N u m(X) \otimes \mathbb{R} \mid x \xi=0\} .
$$

(ii) Define $\mathcal{W}\left(c_{1}, c_{2}\right)$ as the set whose elements consist of $\mathcal{W}^{\xi}$, where $\xi$ is the numerical equivalence class of a divisor $D$ on $X$ such that $\mathcal{O}_{X}\left(D+c_{1}\right)$ is divisible by 2 in $\operatorname{Pic}(X)$, and that

$$
D^{2}<0 ; \quad c_{2}+\frac{D^{2}-c_{1}^{2}}{4}=[Z]
$$

for some locally complete intersection codimension-two cycle $Z$ in $X$.

(iii) A wall of type $\left(c_{1}, c_{2}\right)$ is an element in $\mathcal{W}\left(c_{1}, c_{2}\right)$. A chamber of type $\left(c_{1}, c_{2}\right)$ is a connected component of $C_{X} \backslash \mathcal{W}\left(c_{1}, c_{2}\right)$. A $\mathbb{Z}$-chamber of type $\left(c_{1}, c_{2}\right)$ is the intersection of $\operatorname{Num}(X)$ with some chamber of type $\left(c_{1}, c_{2}\right)$.

(iv) A face of type $\left(c_{1}, c_{2}\right)$ is $F=\mathcal{W}^{\xi} \cap \bar{C}$, where $\mathcal{W}^{\xi}$ is a wall of type $\left(c_{1}, c_{2}\right)$ and $C$ is a chamber of type $\left(c_{1}, c_{2}\right)$.

We say that a wall $\mathcal{W}^{\xi}$ of type $\left(c_{1}, c_{2}\right)$ separates two polarizations $L$ and $L^{\prime}$ if, and only if, $\xi L<0<\xi L^{\prime}$.

Remark 3.3. In [19]; Corollary 2.2.2 and Remark 2.2.6, Qin proves that the moduli space $M_{X, L}\left(2 ; c_{1}, c_{2}\right)$ only depends on the chamber of $L$ and that the study of moduli spaces of rank two vector bundles stable with respect to a polarization lying on walls may be reduced to the study of moduli spaces of rank two vector bundles stable with respect to a polarization lying on $\mathbb{Z}$-chambers. 
Definition 3.4. Let $\xi$ be a numerical equivalence class defining a wall of type $\left(c_{1}, c_{2}\right)$. We define $\mathcal{E}_{\xi}\left(c_{1}, c_{2}\right)$ as the quasi-projective variety parameterizing rank 2 vector bundles $E$ on $X$ given by an extension

$$
0 \rightarrow \mathcal{O}_{X}(D) \rightarrow E \rightarrow \mathcal{O}_{X}\left(c_{1}-D\right) \otimes I_{Z} \rightarrow 0
$$

where $D$ is a divisor with $2 D-c_{1} \equiv \xi$ and $Z$ is a locally complete intersection 0-cycle of length $c_{2}+\left(\xi^{2}-c_{1}^{2}\right) / 4$. Moreover, we require that $E$ is not given by the trivial extension when $\xi^{2}=c_{1}^{2}-4 c_{2}$.

Remark 3.5. By [19]; Theorem 1.2.5, if $L_{1}$ and $L_{2}$ are two ample divisors on $X$ and $E$ is a rank 2 vector bundle on $X$ which is $L_{1}$-stable but $L_{2}$-unstable, then we have $E \in \mathcal{E}_{\xi}\left(c_{1}, c_{2}\right)$ where $\xi$ defines a non-empty wall of type $\left(c_{1}, c_{2}\right)$ separating $L_{1}$ and $L_{2}$ (i.e. $\xi L_{1}<0<\xi L_{2}$; moreover, we can consider the ample divisor $L:=\left(\xi L_{2}\right) L_{1}-\left(\xi L_{1}\right) L_{2}$ on $X$ and we have $L \xi=0)$. More can be said, by [19]; Theorem 1.3.3, given $L_{1}$ and $L_{2}$ two polarizations lying on chambers $C_{1}$ and $C_{2}$, sharing a common wall, we have

$$
M_{L_{1}}\left(2 ; c_{1}, c_{2}\right)=\left(M_{L_{2}}\left(2 ; c_{1}, c_{2}\right) \backslash \sqcup_{\xi} \mathcal{E}_{\xi}\left(c_{1}, c_{2}\right)\right) \sqcup\left(\sqcup_{\xi} \mathcal{E}_{-\xi}\left(c_{1}, c_{2}\right)\right),
$$

where $\xi$ satisfies $\xi L_{1}>0$ and runs over all numerical equivalence classes which define the common wall $\mathcal{W}$.

In the next result, we have summarized well-known properties of some moduli spaces of stable rank two vector bundles on projective surfaces that we will need later on (see for instance [6]; Proposition 3.11).

Proposition 3.6. Let $X$ be a smooth, projective rational surface with effective anticanonical line bundle and let $H$ be an ample line bundle on $X$. Then, the moduli space $M_{X, H}\left(2 ; c_{1}, c_{2}\right)$ of rank two, $H$-stable vector bundles $E$ on $X$ with fixed Chern classes $c_{i}(E)=c_{i}$ is either empty or a smooth irreducible variety of dimension

$$
\operatorname{dim} M_{X, H}\left(2 ; c_{1}, c_{2}\right)=4 c_{2}-c_{1}^{2}-3
$$

For any integer $e \geq 0$, let $X=\mathbb{F}_{e} \cong \mathbb{P}\left(\mathcal{O}_{\mathbb{P}^{1}} \oplus \mathcal{O}_{\mathbb{P}^{1}}(-e)\right)$ be a non singular, Hirzebruch surface. We denote by $C_{0}$ and $F$ the standard basis of $\operatorname{Pic}(X) \cong \mathbb{Z} \oplus \mathbb{Z}$ such that $C_{0}^{2}=-e$, $F^{2}=0$ and $C_{0} F=1$. The canonical divisor is given by

$$
K_{X}=-2 C_{0}-(e+2) F
$$

and it is well known that a divisor $L=a C_{0}+b F$ on $X$ is ample if, and only if, it is very ample, if and only if, $a>0$ and $b>a e$, and that $D=a^{\prime} C_{0}+b^{\prime} F$ is effective if and only if $a^{\prime} \geq 0$ and $b^{\prime} \geq 0([12] \mathrm{V}$, Corollary 2.18).

Given an integer $c_{2}>0$ and $\alpha \in\{0,1\}$, we denote by $M_{L}\left(2 ; C_{0}+\alpha F, c_{2}\right)$ the moduli space of rank two, $L$-stable vector bundles $E$ on $X$ with fixed Chern classes $c_{1}(E)=$ $C_{0}+\alpha F$ and $c_{2}(E)=c_{2}$. For any integer $n, 1 \leq n \leq c_{2}-1$, consider the following ample divisor on $X$

$$
L_{n}:=C_{0}+\left(e+2 c_{2}-\alpha-2 n+1\right) F
$$


Notice that the equivalence class

$$
\xi_{n}:=C_{0}-\left(2 c_{2}-\alpha-2 n\right) F
$$

defines a non-empty wall $\mathcal{W}^{\xi_{n}}$ of type $\left(C_{0}+\alpha F, c_{2}\right)$ which separates the ample divisors $L_{n}$ and $L_{n+1}$. Indeed, $\xi_{n}^{2}=-e-2\left(2 c_{2}-\alpha-2 n\right)<0, \xi_{n}+c_{1}=2\left(C_{0}-\left(c_{2}-\alpha-n\right) F\right)$ is divisible by two in $\operatorname{Pic}(X), c_{2}+\frac{\xi_{n}^{2}-c_{1}^{2}}{4}=n>0$ and

$$
L_{n+1} \xi_{n}=-1<0<1=L_{n} \xi_{n} .
$$

We are led to pose the following problem

Problem 3.7. Determine the difference between the moduli spaces

$$
M_{L_{n}}\left(2 ; C_{0}+\alpha F, c_{2}\right) \text { and } M_{L_{n+1}}\left(2 ; C_{0}+\alpha F, c_{2}\right) .
$$

Remark 3.8. Given an ample line bundle $L=a C_{0}+b F$ on $X$, we can represent $L$ as a point of coordinates $(a, b)$ in the plane. The following picture gives us an idea of the situation we are discussing:

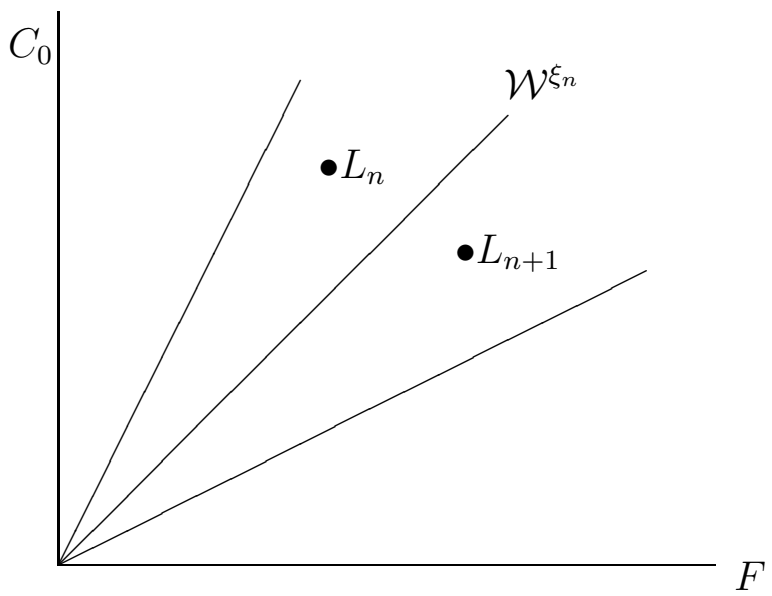

Next result completely solves this problem. We are going to prove that these differences are surprisingly controlled by the following two Brill-Noether loci:

$$
W_{L_{n+1}}^{1}\left(2 ; \overline{c_{1}}, n\right) \subset M_{L_{n+1}}\left(2 ; \overline{c_{1}}, n\right) \quad \text { and } \quad W_{L_{n}}^{1}\left(2 ; \tilde{c_{1}}, n\right) \subset M_{L_{n}}\left(2 ; \tilde{c_{1}}, n\right)
$$

where $\overline{c_{1}}=-C_{0}+\left(2 c_{2}-\alpha-2 n\right) F$ and $\tilde{c_{1}}=C_{0}+\left(\alpha+2 n-2 c_{2}\right) F$.

Theorem 3.9. Let $X=\mathbb{F}_{e}$ be a smooth Hirzebruch surface, $c_{2}>1$ an integer and $\alpha \in\{0,1\}$. For any integer $n, 1 \leq n \leq c_{2}-1$, consider the ample divisor $L_{n}:=$ $C_{0}+\left(e+2 c_{2}-\alpha-2 n+1\right) F$. Then

$$
M_{L_{n}}\left(2 ; C_{0}+\alpha F, c_{2}\right) \cong\left(M_{L_{n+1}}\left(2 ; C_{0}+\alpha F, c_{2}\right) \backslash W_{L_{n+1}}^{1}\left(2 ; \overline{c_{1}}, n\right)\right) \sqcup W_{L_{n}}^{1}\left(2 ; \tilde{c_{1}}, n\right) .
$$


Moreover, the Brill-Noether loci

$$
W_{L_{n+1}}^{1}\left(2 ; \overline{c_{1}}, n\right) \subset M_{L_{n+1}}\left(2 ; \overline{c_{1}}, n\right) \quad \text { and } \quad W_{L_{n}}^{1}\left(2 ; \tilde{c_{1}}, n\right) \subset M_{L_{n}}\left(2 ; \tilde{c_{1}}, n\right)
$$

do have the expected dimension $\rho_{L_{n+1}}^{1}\left(2 ; \overline{c_{1}}, n\right)$ and $\rho_{L_{n}}^{1}\left(2 ; \tilde{c}_{1}, n\right)$, respectively.

Proof. We have already seen that the numerical class

$$
\xi_{n}:=C_{0}-\left(2 c_{2}-\alpha-2 n\right) F
$$

defines a non-empty wall $\mathcal{W}^{\xi_{n}}$ of type $\left(C_{0}+\alpha F, c_{2}\right)$ which separates the ample divisors

$$
L_{n}=C_{0}+\left(e+2 c_{2}-\alpha-2 n+1\right) F \quad \text { and } \quad L_{n+1}=C_{0}+\left(e+2 c_{2}-\alpha-2 n-1\right) F .
$$

Hence, by Remark 3.5 we have:

$$
M_{L_{n}}\left(2 ; C_{0}+\alpha F, c_{2}\right)=\left(M_{L_{n+1}}\left(2 ; C_{0}+\alpha F, c_{2}\right) \backslash \sqcup_{\xi} \mathcal{E}_{\xi}\left(c_{1}, c_{2}\right)\right) \sqcup\left(\sqcup_{\xi} \mathcal{E}_{-\xi}\left(c_{1}, c_{2}\right)\right),
$$

where $\xi$ satisfies $\xi L_{n}>0$ and runs over all numerical equivalence classes which define the common wall $\mathcal{W}^{\xi_{n}}$ separating $L_{n}$ and $L_{n+1}$.

Claim 1: $\xi_{n}$ is the only equivalence class which defines the common wall $\mathcal{W}^{\xi_{n}}$ and verifies $\xi_{n} L_{n}>0$.

Proof of Claim 1: Let $L=a C_{0}+b F \in \mathcal{W}^{\xi_{n}}$ be an ample divisor lying on the common wall defined by $\xi_{n}$. By definition,

$$
0=L \xi_{n}=-a e-a\left(2 c_{2}-\alpha-2 n\right)+b
$$

and thus $L=a C_{0}+a\left(2 c_{2}-\alpha-2 n+e\right)$. Assume there exists a numerical equivalence class $\xi=\sigma C_{0}+\gamma F$ defining the common wall $\mathcal{W}^{\xi_{n}}$ and such that $\xi L_{n}>0$. In particular, since $L=C_{0}+\left(2 c_{2}-\alpha-2 n+e\right) \in \mathcal{W}^{\xi_{n}}$, we have

$$
0=\xi L=\left(\sigma C_{0}+\gamma F\right)\left(C_{0}+\left(2 c_{2}-\alpha-2 n+e\right) F\right)=-\sigma e+\sigma\left(2 c_{2}-\alpha-2 n+e\right)+\gamma
$$

and hence $\gamma=-\sigma\left(2 c_{2}-\alpha-2 n\right)$. On the other hand, since $\xi$ defines a non-empty wall

$$
0 \leq c_{2}+\frac{\xi^{2}-c_{1}^{2}}{4}=c_{2}-\frac{\sigma^{2}}{2}\left(2 c_{2}-\alpha-2 n\right)+\frac{e}{4}\left(1-\sigma^{2}\right)-\frac{\alpha}{2}
$$

which gives us $\sigma= \pm 1$. Finally, $\xi L_{n}>0$ implies $\sigma=1$ and thus $\xi=C_{0}-\left(2 c_{2}-\alpha-2 n\right) F=$ $\xi_{n}$ which proves Claim 1 .

Applying Claim 1, equation (3.2) turns out to be

$$
M_{L_{n}}\left(2 ; C_{0}+\alpha F, c_{2}\right)=\left(M_{L_{n+1}}\left(2 ; C_{0}+\alpha F, c_{2}\right) \backslash \mathcal{E}_{\xi_{n}}\left(c_{1}, c_{2}\right)\right) \sqcup \mathcal{E}_{-\xi_{n}}\left(c_{1}, c_{2}\right) .
$$

Notice that

$$
\begin{aligned}
\tilde{c_{1}} L_{n} & =\left(C_{0}+\left(\alpha+2 n-2 c_{2}\right) F\right)\left(C_{0}+\left(e+2 c_{2}-\alpha-2 n+1\right) F\right) \\
& =1 \\
& >2\left(-4 c_{2}+4 n+2 \alpha-e-4\right) \\
& =2\left(-2 C_{0}-(e+2) F\right)\left(C_{0}+\left(e+2 c_{2}-\alpha-2 n+1\right) F\right) \\
& =2 K_{X} L_{n}
\end{aligned}
$$


and

$$
\begin{aligned}
\overline{c_{1}} L_{n+1} & =\left(-C_{0}+\left(2 c_{2}-\alpha-2 n\right) F\right)\left(C_{0}+\left(e+2 c_{2}-\alpha-2 n-1\right) F\right) \\
& =1 \\
& >2\left(-4 c_{2}+4 n+2 \alpha-e\right) \\
& =2\left(-2 C_{0}-(e+2) F\right)\left(C_{0}+\left(e+2 c_{2}-\alpha-2 n-1\right) F\right) \\
& =2 K_{X} L_{n+1} .
\end{aligned}
$$

Thus by Corollary 2.8 the Brill-Noether stratification of the moduli spaces $M_{L_{n}}\left(2 ; \tilde{c_{1}}, n\right)$ and $M_{L_{n+1}}\left(2 ; \overline{c_{1}}, n\right)$ are defined.

Claim 2: We have:

$$
\begin{aligned}
& \text { (a) } \mathcal{E}_{-\xi_{n}} \cong W_{L_{n}}^{1}\left(2 ; \tilde{c_{1}}, n\right), \\
& \text { (b) } \mathcal{E}_{\xi_{n}} \cong W_{L_{n+1}}^{1}\left(2 ; \overline{c_{1}}, n\right) .
\end{aligned}
$$

Proof of Claim 2: First of all notice that Claim 2 is, by definition, equivalent to have

$$
\begin{gathered}
\left(a^{\prime}\right) \quad \mathcal{E}_{-\xi_{n}} \cong\left\{G \in M_{L_{n}}\left(2 ; C_{0}+\alpha F, c_{2}\right) \mid h^{0}\left(G\left(-\left(c_{2}-n\right) F\right)\right)>0\right\}, \\
\left(b^{\prime}\right) \quad \mathcal{E}_{\xi_{n}} \cong\left\{G \in M_{L_{n+1}}\left(2 ; C_{0}+\alpha F, c_{2}\right) \mid h^{0}\left(G\left(-C_{0}+\left(c_{2}-n-\alpha\right) F\right)\right)>0\right\} .
\end{gathered}
$$

Let us prove $\left(a^{\prime}\right)$. If $E \in \mathcal{E}_{-\xi_{n}}$, then $E$ is given by a non-trivial extension

$$
0 \rightarrow \mathcal{O}_{X}\left(\left(c_{2}-n\right) F\right) \rightarrow E \rightarrow \mathcal{O}_{X}\left(C_{0}-\left(c_{2}-n-\alpha\right) F\right) \otimes I_{Z} \rightarrow 0
$$

where $Z$ is a 0 -dimensional scheme of length $|Z|=c_{2}\left(E\left(-\left(c_{2}-n\right) F\right)\right)=n$. Therefore $h^{0}\left(E\left(-\left(c_{2}-n\right) F\right)\right)>0$. Moreover, it follows from (3.3) that $\mathcal{E}_{-\xi_{n}} \subset M_{L_{n}}\left(2 ; C_{0}+\alpha F, c_{2}\right)$. Now let us see the converse. Let $E \in\left\{G \in M_{L_{n}}\left(2 ; C_{0}+\alpha F, c_{2}\right) \mid h^{0}\left(G\left(-\left(c_{2}-n\right) F\right)\right)>0\right\}$ and we are going to see that $E \in \mathcal{E}_{-\xi_{n}}$. Let $s$ be a non-zero section of $E\left(-\left(c_{2}-n\right) F\right)$ and let $Y$ be its scheme of zeros. Let $D$ be the maximal effective divisor contained in $Y$. Then $s$ can be regarded as a section of $E\left(-\left(c_{2}-n\right) F-D\right)$ and its scheme of zeros has codimension $\geq 2$. Thus, for some effective divisor $D=a C_{0}+b F$ we have a short exact sequence

$$
0 \rightarrow \mathcal{O}_{X}\left(\left(c_{2}-n\right) F+D\right) \rightarrow E \rightarrow \mathcal{O}_{X}\left(C_{0}-\left(c_{2}-n-\alpha\right) F-D\right) \otimes I_{Z} \rightarrow 0 .
$$

By assumption, $E$ is $L_{n}$-stable. Therefore,

$$
\left(\left(c_{2}-n\right) F+D\right) L_{n}<\frac{c_{1}(E) L_{n}}{2}=\frac{\left(C_{0}+\alpha F\right) L_{n}}{2}
$$

which is equivalent to $a\left(2 c_{2}-\alpha-2 n+1\right)+b \leq 0$. Since $D$ is an effective divisor $a, b \geq 0$ and hence the only solution is $a=b=0$. Therefore $D=0$ and $E$ is given by the exact sequence

$$
0 \rightarrow \mathcal{O}_{X}\left(\left(c_{2}-n\right) F\right) \rightarrow E \rightarrow \mathcal{O}_{X}\left(C_{0}-\left(c_{2}-n-\alpha\right) F\right) \otimes I_{Z} \rightarrow 0
$$

which proves that $E \in \mathcal{E}_{-\xi_{n}}$.

Let us now prove $\left(b^{\prime}\right)$. By Remark (3.3), $\mathcal{E}_{\xi_{n}} \subset M_{L_{n+1}}\left(2 ; C_{0}+\alpha F, c_{2}\right)$ and since any $E \in \mathcal{E}_{\xi_{n}}$ is given by a non-trivial extension of type

$$
0 \rightarrow \mathcal{O}_{X}\left(C_{0}-\left(c_{2}-n-\alpha\right) F\right) \rightarrow E \rightarrow \mathcal{O}_{X}\left(\left(c_{2}-n\right) F\right) \otimes I_{Z} \rightarrow 0,
$$

for any $E \in \mathcal{E}_{\xi_{n}}$, we have $h^{0}\left(E\left(-C_{0}+\left(c_{2}-n-\alpha\right) F\right)\right)>0$. Conversely, given

$$
E \in\left\{G \in M_{L_{n+1}}\left(2 ; C_{0}+\alpha F, c_{2}\right) \mid h^{0}\left(G\left(-C_{0}+\left(c_{2}-n-\alpha\right) F\right)\right)>0\right\}
$$


we are going to see that $E \in \mathcal{E}_{\xi_{n}}$. Let $s$ be a non-zero section of $E\left(-C_{0}+\left(c_{2}-n-\alpha\right) F\right)$ and let $Y$ be its scheme of zeros. Let $D$ be the maximal effective divisor contained in $Y$. Then $s$ can be regarded as a section of $E\left(-C_{0}+\left(c_{2}-n-\alpha\right) F-D\right)$ and its scheme of zeros has codimension $\geq 2$. Thus, for some effective divisor $D=a C_{0}+b F$ we have a short exact sequence

$$
0 \rightarrow \mathcal{O}_{X}\left(C_{0}-\left(c_{2}-n-\alpha\right) F+D\right) \rightarrow E \rightarrow \mathcal{O}_{X}\left(\left(c_{2}-n\right) F-D\right) \otimes I_{Z} \rightarrow 0 .
$$

By assumption, $E$ is $L_{n+1}$-stable. Therefore,

$$
\left(C_{0}-\left(c_{2}-n-\alpha\right) F+D\right) L_{n+1}<\frac{c_{1}(E) L_{n+1}}{2}=\frac{\left(C_{0}+\alpha F\right) L_{n+1}}{2},
$$

which is equivalent to $a\left(2 c_{2}-\alpha-2 n-1\right)+b \leq 0$. Since $D$ is an effective divisor this implies that $a=b=0$ and thus $E$ is given by the exact sequence

$$
0 \rightarrow \mathcal{O}_{X}\left(C_{0}-\left(c_{2}-n-\alpha\right) F\right) \rightarrow E \rightarrow \mathcal{O}_{X}\left(\left(c_{2}-n\right) F\right) \otimes I_{Z} \rightarrow 0
$$

which proves that $E \in \mathcal{E}_{\xi_{n}}$.

Putting together Claim 2 and (3.3) we obtain

$$
M_{L_{n}}\left(2 ; C_{0}+\alpha F, c_{2}\right) \cong\left(M_{L_{n+1}}\left(2 ; C_{0}+\alpha F, c_{2}\right) \backslash W_{L_{n+1}}^{1}\left(2 ; \overline{c_{1}}, n\right)\right) \sqcup W_{L_{n}}^{1}\left(2 ; \tilde{c_{1}}, n\right) .
$$

Finally let us see that the dimensions of the Brill-Noether loci $W_{L_{n}}^{1}\left(2 ;{\tilde{c_{1}}}_{1}, n\right)$ and $W_{L_{n+1}}^{1}\left(2 ; \overline{c_{1}}, n\right)$ coincide with the expected one. To this end, by Claim 2, it suffices to prove that

$$
\begin{aligned}
& \text { (i) } \operatorname{dim}\left(\mathcal{E}_{-\xi_{n}}\right)=\rho_{L_{n}}^{1}\left(2 ; \tilde{c_{1}}, n\right), \\
& \text { (ii) } \operatorname{dim}\left(\mathcal{E}_{\xi_{n}}\right)=\rho_{L_{n+1}}^{1}\left(2 ; \overline{c_{1}}, n\right) \text {. }
\end{aligned}
$$

By construction,

$$
\begin{aligned}
\operatorname{dim} \mathcal{E}_{-\xi_{n}} & =\operatorname{ext}^{1}\left(I_{Z}\left(C_{0}-\left(c_{2}-n-\alpha\right) F\right), \mathcal{O}_{X}\left(\left(c_{2}-n\right) F\right)\right)+2|Z|-h^{0} E\left(-\left(c_{2}-n\right) F\right) \\
& =h^{1}\left(I_{Z}\left(-C_{0}-\left(2 c_{2}-2 n-\alpha+e+2\right) F\right)\right)+2|Z|-h^{0} E\left(-\left(c_{2}-n\right) F\right)
\end{aligned}
$$

being $E \in \mathcal{E}_{-\xi_{n}}$. Since $h^{i}\left(\mathcal{O}_{X}\left(-C_{0}-\left(2 c_{2}-2 n-\alpha+e+2\right) F\right)\right)=0$, for $i=0$, 1 , from the cohomological exact sequence associated to

$$
\begin{gathered}
0 \rightarrow I_{Z}\left(-C_{0}-\left(2 c_{2}-2 n-\alpha+e+2\right) F\right) \rightarrow \mathcal{O}_{X}\left(-C_{0}-\left(2 c_{2}-2 n-\alpha+e+2\right) F\right) \\
\rightarrow \mathcal{O}_{Z}\left(-C_{0}-\left(2 c_{2}-2 n-\alpha+e+2\right) F\right) \rightarrow 0
\end{gathered}
$$

we deduce that

$h^{1}\left(I_{Z}\left(-C_{0}-\left(2 c_{2}-2 n-\alpha+e+2\right) F\right)\right)=h^{0}\left(\mathcal{O}_{Z}\left(-C_{0}-\left(2 c_{2}-2 n-\alpha+e+2\right) F\right)\right)=|Z|$.

For any $E \in \mathcal{E}_{-\xi_{n}}$, we have $h^{0} E\left(-\left(c_{2}-n\right) F\right)=1$ and $c_{2}\left(E\left(-\left(c_{2}-n\right) F\right)\right)=n=|Z|$.

Putting altogether we get

$$
\operatorname{dim} \mathcal{E}_{-\xi_{n}}=3 n-1 .
$$

On the other hand, by Riemann-Roch theorem,

$$
\begin{aligned}
\chi\left(2 ; \tilde{c_{1}}, n\right) & =2+\frac{\left(\tilde{c_{1}}\right)\left(2 C_{0}+(e+2) F\right)}{2}+\frac{\left(\tilde{c_{1}}\right)^{2}}{2}-n \\
& =2+\frac{\left(C_{0}+\left(\alpha+2 n-2 c_{2}\right) F\right)\left(2 C_{0}+(e+2) F\right)}{2}+\frac{\left(C_{0}+\left(\alpha+2 n-2 c_{2}\right) F\right)^{2}}{2}-n \\
& =3 n-4 c_{2}+2 \alpha-e+3
\end{aligned}
$$


and by Proposition $3.6 \operatorname{dim} M_{L_{n}}\left(2 ; \tilde{c_{1}}, n\right)=4 c_{2}-(2 \alpha-e)-3$. Therefore,

$$
\rho_{L_{n}}^{1}\left(2 ; \tilde{c_{1}}, n\right)=\operatorname{dim} M_{L_{n}}\left(2 ; \tilde{c_{1}}, n\right)-1\left(1-\chi\left(2, \tilde{c_{1}}, n\right)\right)=3 n-1
$$

which proves $(i)$.

Let us now prove $(i i)$. By construction,

$$
\begin{aligned}
\operatorname{dim} \mathcal{E}_{\xi_{n}} & =\operatorname{ext}^{1}\left(I_{Z}\left(\left(c_{2}-n\right) F\right), \mathcal{O}_{X}\left(C_{0}-\left(c_{2}-n-\alpha\right) F\right)+2|Z|-h^{0} E\left(-C_{0}+\left(c_{2}-n-\alpha\right) F\right)\right. \\
& =h^{1}\left(I_{Z}\left(-3 C_{0}+\left(2 c_{2}-2 n-\alpha-e-2\right) F\right)\right)+2|Z|-h^{0} E\left(-C_{0}+\left(c_{2}-n-\alpha\right) F\right)
\end{aligned}
$$

being $E \in \mathcal{E}_{\xi_{n}}$. Since $h^{i}\left(\mathcal{O}_{X}\left(-3 C_{0}+\left(2 c_{2}-2 n-\alpha-e-2\right) F\right)\right)=0$, for $i=0,2$, $h^{1}\left(\mathcal{O}_{X}\left(-3 C_{0}+\left(2 c_{2}-2 n-\alpha-e-2\right) F\right)\right)=-\chi\left(\mathcal{O}_{X}\left(-3 C_{0}+\left(2 c_{2}-2 n-\alpha-e-2\right) F\right)\right)=-\chi$ and by Riemann-Roch theorem

$$
\begin{aligned}
\chi & =1+\frac{\left(-3 C_{0}+\left(2 c_{2}-2 n-\alpha-e-2\right) F\right)\left(2 C_{0}+(e+2) F\right)}{2}+\frac{\left(-3 C_{0}+\left(2 c_{2}-2 n-\alpha-e-2\right) F\right)^{2}}{2} \\
& =4 c_{2}-4 n-2 \alpha-2+e .
\end{aligned}
$$

From the cohomological exact sequence associated to

$$
\begin{gathered}
0 \rightarrow I_{Z}\left(-3 C_{0}+\left(2 c_{2}-2 n-\alpha-e-2\right) F\right) \rightarrow \mathcal{O}_{X}\left(-3 C_{0}+\left(2 c_{2}-2 n-\alpha-e-2\right) F\right) \\
\rightarrow \mathcal{O}_{Z}\left(-3 C_{0}+\left(2 c_{2}-2 n-\alpha-e-2\right) F\right) \rightarrow 0
\end{gathered}
$$

we deduce that

$$
\begin{aligned}
h^{1}\left(I_{Z}\left(-3 C_{0}+\left(2 c_{2}-2 n-\alpha-e-2\right) F\right)\right) & =h^{0}\left(\mathcal{O}_{Z}\left(-C_{0}-\left(2 c_{2}-2 n-\alpha+e+2\right) F\right)\right) \\
& +h^{1}\left(\mathcal{O}_{X}\left(-3 C_{0}+\left(2 c_{2}-2 n-\alpha-e-2\right) F\right)\right) \\
& =|Z|+4 c_{2}-4 n-2 \alpha-2+e .
\end{aligned}
$$

For any $E \in \mathcal{E}_{\xi_{n}}$, we have $h^{0} E\left(-C_{0}+\left(c_{2}-n-\alpha\right) F\right)=1$ and $c_{2}\left(E\left(-C_{0}+\left(c_{2}-n-\alpha\right) F\right)=\right.$ $n=|Z|$. Putting altogether we get

$$
\operatorname{dim} \mathcal{E}_{\xi_{n}}=4 c_{2}-n+e-2 \alpha-3 .
$$

On the other hand, by Riemann-Roch theorem,

$$
\chi\left(2 ; \overline{c_{1}}, n\right)=2+\frac{\left(\overline{c_{1}}\right)\left(2 C_{0}+(e+2) F\right)}{2}+\frac{{\overline{c_{1}}}^{2}}{2}-n=1-n,
$$

and by Proposition $3.6 \operatorname{dim} M_{L_{n}}\left(2 ; \overline{c_{1}}, n\right)=4 c_{2}-(2 \alpha-e)-3$. Thus,

$$
\rho_{L_{n+1}}^{1}\left(2 ; \overline{c_{1}}, n\right)=\operatorname{dim} M_{L_{n+1}}\left(2 ; \overline{c_{1}}, n\right)-1\left(1-\chi\left(2 ; \overline{c_{1}}, n\right)\right)=4 c_{2}-n+e-2 \alpha-3
$$

which proves $(i i)$.

\section{BRILl-Noether LOCI AND InSTANTON BUNDLES}

In this section, we will address the three problems posed in section two for the case of mathematical instanton bundles on $\mathbb{P}^{3}$. More precisely, we will prove that the BrillNoether locus of instanton bundles on $\mathbb{P}^{3}$ is non-empty if and only if the corresponding generalized Brill-Noether number is non-negative and in this case the Brill-Noether locus is a smooth irreducible variety of the expected dimension.

Let $M I(n)$ be the moduli space of mathematical instanton bundles $E$ over $\mathbb{P}^{3}$ with $c_{1}(E)=2$ and $c_{2}(E)=n$ and verifying the instanton condition $H^{1}(E(-3))=0$. It is known that $M I(n)$ is non-singular and irreducible for $n \leq 6$ (see [2] for $n=2$, [13] for $n=3$, [10] for $n=4$, [3] and [17] for $n=5$ and [5] for $n=6$ ) and it has been 
conjectured that $M I(n)$ is non-singular and irreducible. The only known component $M I_{0}(n)$ of $M I(n)$ is the one made of vector bundles which are generalizations of (a twist of) the ones associated to $n+1$ skew lines in $\mathbb{P}^{3}$ ('t Hooft bundles) and we know that $M I_{0}(n)$ is a generically smooth variety of dimension $8 n-11$.

Since for any instanton bundle $E \in M I_{0}(n)$, we have $H^{i}\left(\mathbb{P}^{3}, E\right)=0$ for $i \geq 2$, Theorem 2.3 applies and the Brill-Noether stratification of $M I_{0}(n)$ is well defined. So, for any $k \geq 1$, we can study the Brill-Noether loci

$$
W^{k}:=\left\{E \in M I_{0}(n) \mid h^{0}(E) \geq k\right\} \subset M I_{0}(n) .
$$

We have:

Proposition 4.1. Assume $n>13$. With the above notations we have

(i) $W^{k} \neq \emptyset$ if and only if $\rho^{k}(2 ; 2, n) \geq 0$ if and only if $k<3$.

(ii) $W^{1}$ is a smooth rational irreducible quasi-projective variety of the expected dimension $5 n-1$.

(iii) $W^{2}$ is a smooth rational irreducible quasi-projective variety of the expected dimension $2 n+7$.

Proof. (i) Let us first compute $\rho^{k}(2 ; 2, n)$. To this end, we take $E$ a mathematical instanton bundle with Chern classes $\left(c_{1}(E), c_{2}(E)\right)=(2, n)$. It is well-known that $E(-1)$ is the cohomology bundle of a monad of the following type

$$
0 \longrightarrow \mathcal{O}_{\mathbb{P}^{3}}(-1)^{n-1} \stackrel{\alpha}{\longrightarrow} \mathcal{O}_{\mathbb{P}^{3}}^{2 n} \stackrel{\beta}{\longrightarrow} \mathcal{O}_{\mathbb{P}^{3}}(1)^{n-1} \longrightarrow 0 .
$$

Hence we have two exact sequences

$$
0 \longrightarrow \mathcal{K}=\operatorname{ker}(\beta) \rightarrow \mathcal{O}_{\mathbb{P}^{3}}^{2 n} \stackrel{\beta}{\longrightarrow} \mathcal{O}_{\mathbb{P}^{3}}(1)^{n-1} \longrightarrow 0
$$

and

$$
0 \longrightarrow \mathcal{O}_{\mathbb{P}^{3}}(-1)^{n-1} \stackrel{\alpha}{\longrightarrow} \mathcal{K} \rightarrow E(-1) \longrightarrow 0
$$

which allows us to compute $\chi(E)$. Indeed,

$$
\chi(E)=\chi(\mathcal{K}(1))-(n-1)=2 n \chi\left(\mathcal{O}_{\mathbb{P}^{3}}(1)\right)-(n-1) \chi\left(\mathcal{O}_{\mathbb{P}^{3}}(2)\right)-(n-1)=-3 n+11 .
$$

Since $\operatorname{dim} M I_{0}(n)=8 n-11$, we deduce that

$$
\begin{aligned}
\rho^{k}(2 ; 2, n) & =\operatorname{dim} M I_{0}(n)-k(k-\chi(E)) \\
& =8 n-11-k(k+3 n-11) \\
& =n(8-3 k)+k(11-k)-11 .
\end{aligned}
$$

So, $\rho^{1}(2 ; 2, n)=5 n-1, \rho^{2}(2 ; 2, n)=2 n+7$ and $\rho^{k}(2 ; 2, n)<0$ for $k \geq 3$ provided $n \geq 14$. By a result in [4], for any $E \in M I_{0}(n), h^{0}(E) \leq 2$. On the other hand, any instanton bundle $E$ associated to $n+1$ general skew lines in $\mathbb{P}^{3}$ satisfies $h^{0}(E)=1$ ('t Hooft bundles) and any instanton bundle $E$ associated to $n+1$ skew lines on a smooth quadric $Q$ in $\mathbb{P}^{3}$ satisfies $h^{0}(E)=2$ (special 't Hooft bundles). Putting altogether we have $W^{k} \neq \emptyset$ if and only if $k \leq 2$, if and only if $\rho^{k}(2 ; 2, n)>0$.

(ii) and (iii) The expected dimensions are $\rho^{1}(2 ; 2, n)=5 n-1$ and $\rho^{2}(2 ; 2, n)=2 n+7$. Hence, the results follows from the fact that $W^{1}$ (resp. $W^{2}$ ) is nothing but the variety of 't Hooft bundles (resp. special' t Hooft bundles) and we well-known that the variety 
of 't Hooft bundles (resp. special ' t Hooft bundles) is a smooth, irreducible and rational variety of dimension $5 n-1$ (resp. $2 n+7$ ).

\section{REFERENCES}

[1] E. Arbarello; M. Cornalba; P.A. Griffiths; J. Harris, Geometry of algebraic curves. Vol. I. Grundlehren der Mathematischen Wissenschaften [Fundamental Principles of Mathematical Sciences], 267. Springer-Verlag, New York, 1985

[2] W. Barth Some properties of stable rank 2 vector bundles on $\mathbf{P}^{n}$ Math. Ann. 226 (1977) 125-150.

[3] W. Barth Irreducibility of the space of mathematical instanton bundles with rank 2 and $c_{2}=4$ Math. Ann., 258 (1981) 81-106.

[4] W. Böhmer, G. Trautman, Special instanton bundles and Poncelet curves, in Singularities, representations of algebras and vector bundles, Proceedings Lambrecht 1985.

[5] I. Coandă; A. Tikhomirov; G. Trautmann, Irreducibility and smoothness of the moduli space of mathematical 5-instantons over $\mathbb{P}^{3}$. International J. of Math. 14 (2003), 1-45.

[6] L. Costa; R.M. Miró-Roig On the rationality of moduli spaces of vector bundles on Fano surfaces, J. Pure Applied Algebra, 137, (1999), 199-220.

[7] L. Costa; R.M. Miró-Roig, Rationality of moduli spaces of vector bundles on Hirzebruch surfaces, J. reine angew. Math., 159, (1999), 151-166.

[8] L. Costa; R.M. Miró-Roig, Moduli spaces of vector bundles on higher dimensional varieties, Michigan Math. J. 49 (2001), 605-620.

[9] L. Costa; R.M. Miró-Roig, Rationality of moduli spaces of vector bundles on rational surfaces, Nagoya Math. J. 165 (2002), 43-69.

[10] G. Ellingsrud and S.A. Stromme, Stable rank 2 bundles on $\mathbf{P}^{3}$ with $c_{1}=0$ and $c_{2}=3$. Math. Ann. 255 (1991) 123-135.

[11] L. Göttsche, A, Hirschowitz, Weak Brill-Noether for vector bundles on the projective plane. Algebraic geometry (Catania, 1993/Barcelona, 1994), 63-74, Lecture Notes in Pure and Appl. Math., 200, Dekker, New York, (1998).

[12] R. Hartshorne, Algebraic Geometry, GTM 52.

[13] R. Hartshorne, Stable vector bundles of rank 2 on $\mathbf{P}^{3}$ Math. Ann. 238 (1978) 229-280.

[14] R. Hartshorne, A. Hirschowitz, Cohomology of a general instanton bundle, Ann. Sci. École Norm. Sup. (4) 15 (1982) 365-390.

[15] M. Leyenson, On the Brill-Noether theory for K3 surfaces, Preprint arXiv:math/0511659.

[16] M. Leyenson, On the Brill-Noether theory for K3 surfaces, II, Preprint arXiv:math/0602358.

[17] J. Le Potier, Sur l'espace de modules des fibrés de Yang et Mills Prog. Math. 37, (1983) 65-137.

[18] C. Okonek and H. Spindler, Mathematical Instanton bundles on $\mathbb{P}^{2 n+1}$, Crelle J., 364 (1986), 35-50.

[19] Z. Qin, Equivalence classes of polarizations and moduli spaces of sheaves, J. Diff. Geom. 37 (1993), 397-415.

Facultat de Matemàtiques, Departament d'Algebra i Geometria, Gran Via de les Corts Catalanes 585, 08007 Barcelona, SPAin

E-mail address: costa@ub.edu, miro@ub.edu 